\title{
Clinical Outcomes with a New Model of Extended Depth of Focus Intraocular Lens
}

\author{
Francisco Poyales, Nuria Garzón*, Concepción Poyales, Blanca Poyales \\ IOA Madrid Innova Ocular, Madrid, Spain \\ Email: ${ }^{\star}$ garzon@ioamadrid.com
}

How to cite this paper: Poyales, F., Garzón, N., Poyales, C. and Poyales, B. (2018) Clinical Outcomes with a New Model of Extended Depth of Focus Intraocular Lens. Open Journal of Ophthalmology, 8, 161-170.

https://doi.org/10.4236/ojoph.2018.83020

Received: July 16, 2018

Accepted: August 12, 2018

Published: August 15, 2018

Copyright $\odot 2018$ by authors and Scientific Research Publishing Inc. This work is licensed under the Creative Commons Attribution International License (CC BY 4.0).

http://creativecommons.org/licenses/by/4.0/

cc) (7) Open Access

\begin{abstract}
Purpose: To evaluate the early clinical outcomes of a new diffractive extended depth-of-focus (EDOF) intraocular lens (IOL). Methods: Prospective case series enrolling patients undergoing cataract surgery with implantation of the EDOF IOL AT LARA (Carl Zeiss Meditec, Jena, Germany). Distance, intermediate and near visual acuity were evaluated during a follow-up of 1 month. Likewise, defocus curve, binocular mesopic contrast sensitivity, perception of photic phenomena, patient satisfaction and spectacle independence were assessed. Results. The study enrolled 38 eyes of 19 patients with a mean age of 69.5 years. A significant reduction of manifest refraction and improvement in corrected distance visual acuity (CDVA) was observed with surgery ( $\mathrm{p}<$ 0.001). All eyes achieved a 1-month postoperative binocular CDVA of 20/20 or better. Likewise, all eyes achieved a DCIVA of 20/30 or better at 1 month postoperatively, and a total of $92.1 \%$ achieved DCNVA of $20 / 40$ or better. Concerning refractive predictability, $97.4 \%$ of eyes had a 1-month postoperative spherical equivalent within $\pm 0.50 \mathrm{D}$. The level of patient satisfaction with distance, intermediate and near vision was high, with $95 \%$ of patients reporting that they would have the same surgical procedure with the same IOL again. Difficulties in night driving or associated to halos were low. A total of $89 \%$ of patients reported to be spectacle independent postoperatively. Conclusions: The EDOF IOL AT LARA is able to provide a functional restoration of the visual function across distances after cataract surgery, with very high levels of patient satisfaction and minimal incidence of photic phenomena.
\end{abstract}

\section{Keywords}

AT LARA, EDOF, Visual Performance, Visual Symptoms, Patient Satisfaction

\section{Introduction}

In the last years, a new concept of presbyopia-correcting intraocular lenses 
(IOLs) has been developed and widely expanded in clinical practice, the extended depth of focus (EDOF) IOL technology [1]. This type of implants is aimed at providing functional vision at different distances while minimizing the incidence of photic phenomena [1], as the presence of secondary out-of-focus images corresponding to different foci is avoided due to the presence of a continuous range of focus [2]. In other words, no clearly differentiated foci are generated by EDOF IOLs as it is the case with multifocal IOLs [3]. It should be considered that photic phenomena are one of the main causes of dissatisfaction after cataract surgery with implantation of multifocal IOLs [4] and one of the main reasons leading surgeons to multifocal IOL explantation [5].

The main basis of EDOF technology is the use of an optical design providing a more reduced near addition of the IOL while maintaining a continuous range of functional vision across intermediate and near distances. This can be achieved by means of a diffractive [6] [7] or a refractive platform [6] [8] aimed at controlling the level of spherical or coma aberration. Different studies have shown the clinical performance of EDOF IOLs, reporting good visual and contrast sensitivity outcomes, with high levels of patient satisfaction [9]-[14]. Concerning photic phenomena outcomes, whereas some comparative trials have found no significant differences between multifocal and EDOF IOLs [9] [11] [12], others have found a lower incidence of photic phenomena with EDOF designs compared to trifocal IOLs [13]. The aim of the current study was to evaluate the clinical outcomes of a new model of diffractive EDOF IOL in terms of visual acuity across different distances, contrast sensitivity, photic phenomena and patient satisfaction.

\section{Methods}

\subsection{Patients}

This prospective case series enrolled patients undergoing cataract surgery with implantation of the EDOF IOL AT LARA (Carl Zeiss Meditec, Jena, Germany). Inclusion criteria were cataract eyes with no comorbidity, availability, willingness and sufficient cognitive awareness to comply with examination procedures, and signed informed consent. Exclusion criteria were irregular astigmatism, corneal astigmatism of more than $1.00 \mathrm{D}$, patient age below 40 years, difficulty for cooperation (distance from their home, general health condition), acute or chronic disease or illness that would increase risk or confound study results (e.g. diabetes mellitus with retinopathy, immunocompromised, glaucoma etc...), any ocular comorbidity, history of ocular trauma or prior ocular surgery including refractive procedures, capsule or zonular abnormalities that may affect postoperative centration or tilt of the lens (e.g. pseudoexfoliation syndrome, chronic Uveitis, Marfan's syndrome), pupil abnormalities (non-reactive, tonic pupils, abnormally shaped pupils or pupils that do not dilate under mesopic/scotopic conditions), age-related macular degeneration (AMD) suspicious eyes, and/or complicated surgery. All patients were informed about the study and provided 
informed consent to undergo the clinical examinations in accordance with the tenets of the Declaration of Helsinki. The study received the approval of the local ethics committee.

\subsection{Examinations}

All patients underwent a comprehensive preoperative ophthalmological examination including measurement of uncorrected and corrected distance visual acuity (UDVA and CDVA), keratometry, optical biometry (IOLMaster, Carl Zeiss Meditec AG, Germany), manifest refraction, biomicroscopy, Goldmann applanation tonometry, and dilated fundoscopy. Postoperatively, patients were evaluated the day after surgery, and at 1 week and 1 month after surgery. At these two postoperative visits, the following clinical tests were performed: measurement of monocular UDVA and CDVA, measurement of monocular distance-corrected near (DCNVA, $40 \mathrm{~cm}$ ) and intermediate visual acuity (DCIVA) $(80 \mathrm{~cm})$, and manifest refraction. Likewise, at 1 month postoperatively, a monocular distance-corrected defocus curve was obtained in all cases, including levels of defocus from +2 to $-4 \mathrm{D}$ in steps of $0.50 \mathrm{D}$ as well as a measurement of binocular contrast sensitivity under mesopic conditions with Functional Acuity Contrast Test charts (CST 1800 system, Vision Science Research). Patient satisfaction and quality of life were determined by means of a questionnaire, which was completed by the patients at the last follow-up visit. Questions included satisfaction about: adaption between photopic and mesopic conditions, ability to find the correct distance, night driving, vision during the day, halos, adaption between far and near vision and vice versa, as well as general satisfaction for distance, intermediate and near vision, and overall satisfaction. Patients were also asked whether they would perform the same surgery again and whether they need to wear glasses for the daily tasks. Each subscale score was converted to a score between 0 and 5, with higher scores indicating better results. Finally, halometry was also measured at this visit.

\subsection{Surgical Technique}

An experienced surgeon (FP) performed all surgeries using a standard technique of sutureless phacoemulsification. Topical anaesthesia was initially administered, and pharmacologic mydriasis was induced. Once pupillary dilation was achieved, the surgical procedure was initiated with a clear cornea microincision of around $2.2 \mathrm{~mm}$ with a diamond knife and a paracentes is $60^{\circ}-90^{\circ}$ clockwise from the main incision. The IOL was implanted through the main incision using a specific injector. Postoperative pharmacological treatment consisted of a combination of antibiotic and steroidal anti-inflammatory drops. Likewise, non-steroidal anti-inflammatory drops were prescribed to prevent macular edema.

\subsection{Statistical Analysis}

Data analysis was performed using the software SPSS for Windows version 19.0 
(IBM, Armonk, NY, USA). Normality of data samples was evaluated by means of the Kolmogorov-Smirnov test. When parametric analysis was possible, the Student $t$ test for paired data was used to compare the results between consecutive visits. When parametric analysis was not possible, the Wilcoxon test was used to compare the analyzed parameters between visits. For all statistical tests, a p-value of less than 0.05 was considered as statistically significant.

\section{Results}

The sociodemographic and clinical characteristics of the patients is shown in Table 1 .

\subsection{Visual and Refractive Outcomes}

Table 2 summarizes the postoperative visual and refractive outcomes in the analysed sample. As shown, a significant reduction of manifest refraction and a significant improvement in CDVA was observed with surgery $(\mathrm{p}<0.001)$. All eyes achieved a 1-month postoperative binocular CDVA of 20/20 or better (Figure 1). Likewise, all eyes achieved a DCIVA of 20/30 or better at 1 month postoperatively, and a total of $92.1 \%$ (35) achieved DCNVA of 20/40 or better. Concerning refractive predictability, a total of $97.4 \%$ of eyes had a 1-month postoperative spherical equivalent within $\pm 0.50 \mathrm{D}$. Figure 1 shows the mean binocular defocus curve at 1 month after surgery. As shown, a distance-corrected visual acuity over $0.2 \log$ MAR was achieved for defocus levels up to $-2 \mathrm{D}$.

\subsection{Quality of Vision and Patient Satisfaction Outcomes}

Figure 2 shows the mean mesopic monocular contrast sensitivity function evaluated at 1 month after surgery. The mean halo score obtained by halometry was 0.81 (SD: 0.11 ; median: 0.85; range: 0.56 to 0.97 ). Table 3 shows the mean scores of different aspects of quality of vision and patient satisfaction using the subjective questionnaire. Good scores were obtained for all aspects, with the lowest scores associated to night driving and halos. A total of 95\% (18) of the

Table 1. Sociodemographic and clinical characteristics of the patients.

\begin{tabular}{cc}
\hline \multicolumn{1}{c}{ Parameter } & Value \\
\hline $\begin{array}{c}\text { No. of patients } \\
\text { No. of eyes }\end{array}$ & 19 \\
Mean age/median age/age range & $69.5 \pm 6.3$ years; median: 69.0 years; range: \\
Gender distribution & 57 to 83 years \\
Mean preoperative axial length & $23.3 \pm 0.9$ mm; median: 23.3 ; range: 21.8 to $25.1 \mathrm{~mm}$ \\
Mean preoperative anterior chamber depth & $2.9 \pm 0.3$ mm; median: 2.8 ; range: 2.5 to 3.5 mm \\
Mean preoperative flattest keratometry & $43.1 \pm 1.6 \mathrm{D}$; median: 43.0 ; range: 39.4 to $46.2 \mathrm{D}$ \\
Mean preoperative steepest keratometry & $43.8 \pm 1.7 \mathrm{D}$; median: 43.6 ; range: 40.1 to $46.9 \mathrm{D}$ \\
\hline
\end{tabular}


Table 2. Postoperative visual acuity and subjective refraction in the analyzed sample.

\begin{tabular}{ccccc}
\hline & \multicolumn{4}{c}{ Mean (SD) Median (Range) } \\
\cline { 2 - 5 } & Preoperative & 1 week postop & 1 month postop & P-value \\
\hline \multirow{2}{*}{ LogMAR UDVA } & --- & $0.09(0.13)$ & $0.08(0.10)$ & -- \\
& $0.09(0.10)$ & $0.03(0.00$ to 0.52$)$ & $0.03(-0.04$ to 0.30$)$ & \\
LogMAR CDVA & $0.04(0.00$ to 0.38$)$ & $0.00(0.00$ to 0.16$)$ & $0.00(-0.04$ to 0.05$)$ & $<0.001$ \\
& $1.73(1.22)$ & $-0.17(0.39)$ & $-0.17(0.39)$ & $<0.001$ \\
Sphere (D) & $1.75(-1.75$ to 4.00$)$ & $0.00(-1.00$ to 0.75$)$ & $0.00(-1.00$ to 0.75$)$ & \\
& $-0.78(0.50)$ & $-0.20(0.37)$ & $-0.16(0.34)$ & $<0.001$ \\
Cylinder (D) & $-0.75(-1.75$ to 0.00$)$ & $0.00(-1.25$ to 0.00$)$ & $0.00(-1.00$ to 0.00$)$ & \\
& $1.44(1.11)$ & $-0.12(0.32)$ & $-0.16(0.39)$ & $<0.001$ \\
SE (D) & $1.38(-1.75$ to 3.50$)$ & $0.00(-1.00$ to 0.75$)$ & $0.00(-1.25$ to 0.75$)$ & \\
& - & $0.30(0.09)$ & $0.30(0.08)$ & -- \\
LogMAR DCNVA & --- & $0.30(0.00$ to 0.50$)$ & $0.30(0.00$ to 0.50$)$ & \\
& & $0.14(0.06)$ & $0.14(0.07)$ & -- \\
LogMAR DCIVA & -- & $0.10(0.00$ to 0.30$)$ & $0.10(0.00$ to 0.30$)$ & \\
\hline
\end{tabular}

Table 3. Quality of vision and patient satisfaction outcomes obtained using a subjective questionnaire at 1 month after surgery (score from $0=$ worst score to $5=$ best score).

\begin{tabular}{cc}
\hline Categories & $\begin{array}{c}\text { Mean (SD) } \\
\text { Median (Range) }\end{array}$ \\
\hline General satisfaction with distance vision & $4.7(0.6)$ \\
General satisfaction with near vision & $5(3$ to 5) \\
& $4.3(0.7)$ \\
General satisfaction with intermediate vision & $4(3$ to 5) \\
& $4.6(0.7)$ \\
Adaptation between photopic and mesopic conditions & $5(3$ to 5) \\
& $4.2(1.0)$ \\
Ability to find the correct distance & $5(2$ to 5$)$ \\
& $4.9(0.3)$ \\
Night driving & $5(4$ to 5) \\
Vision during the day & $3.8(0.8)$ \\
& $4(3$ to 5) \\
Difficulties associated to halos & $4.7(0.8)$ \\
& $5(2$ to 5) \\
& $3.5(1.2)$ \\
Adaptation between distance and near visual acuity and vice versa & $4(1$ to 5) \\
& $4.4(0.8)$ \\
Overall satisfaction & $5(3$ to 5) \\
\end{tabular}

patients reported that would have the same surgical procedure with the same IOL again. Likewise, and $89 \%$ (17) of the patients reported to be spectacle independent postoperatively.

\section{Discussion}

This is one of the first clinical studies reporting the clinical outcomes obtained with the EDOF IOL AT LARA, which is based on a diffractive platform. Distance visual acuity is excellent with this EDOF IOL, with a 1-month postoperative UDVA and CDVA of $0.08 \pm 0.10$ and $0.00 \pm 0.02$, respectively. Therefore, 


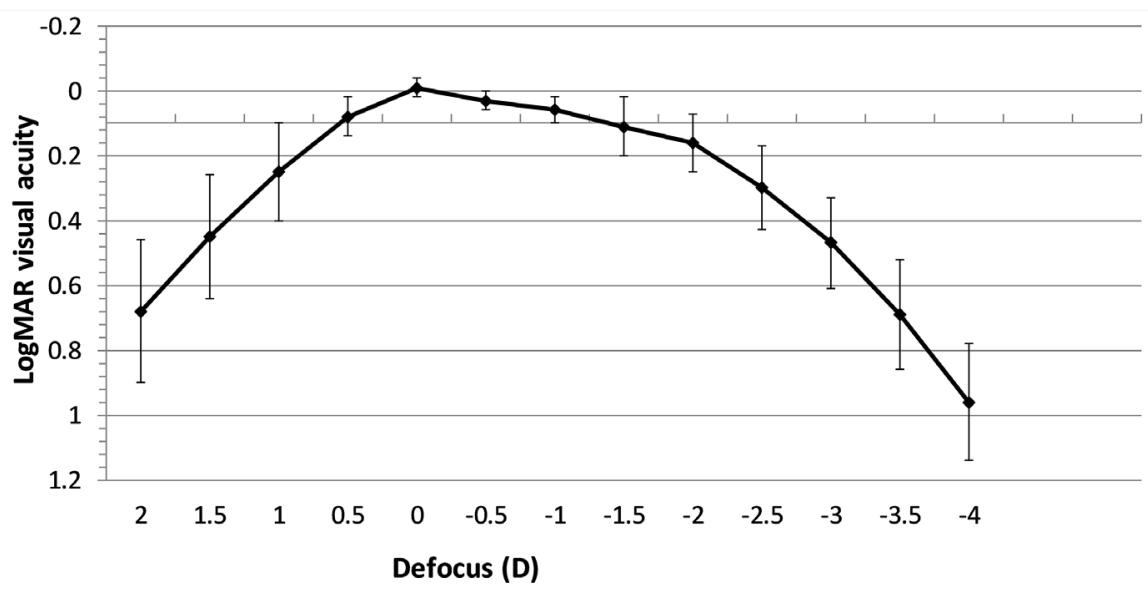

Figure 1. Mean monocular distance-corrected defocus curves at 1 month after surgery.

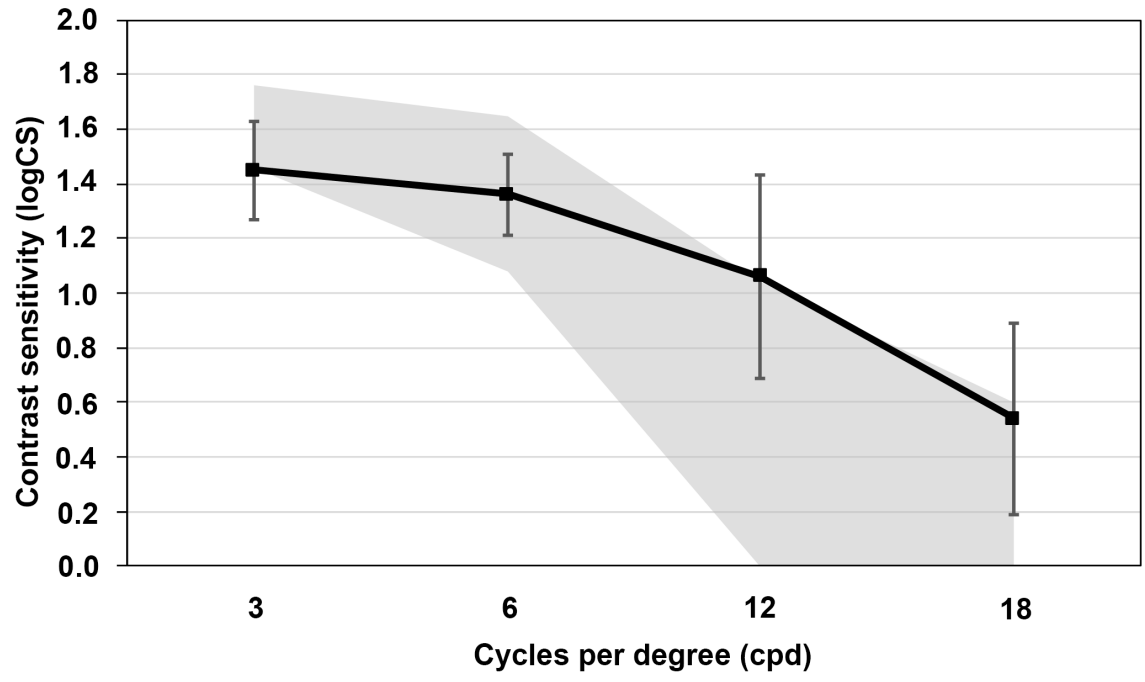

Figure 2. Mean mesopic binocular contrast sensitivity function at 1 month after surgery.

the diffractive platform of this EDOF IOL does not impair distance quality of vision, which is also reflected by the high levels of general satisfaction with distance vision. This is also consistent with the high mesopic contrast sensitivity measured postoperatively with this IOL, which is similar to that reported for patients implanted with monofocal IOLs [9] [15]. Likewise, the distance vision and contrast sensitivity outcomes obtained with the evaluated EDOF IOL is consistent with that reported for other types of EDOF IOLs [9] [10] [11] [12] [13] [15]-[21]. A mean decimal UDVA of 0.95 was reported in the sample of 411 patients implanted with the diffractive-based EDOF IOL Tecnis Symfony (Johnson \& Johnson Vision, USA) in the Concerto multicenter study, with a median patient satisfaction score of 9.0 (scale, 0 to 10) [19]. Pedrotti and colleagues [20] compared the results of the same Symfony EDOF IOL with those obtained with a monofocal aspheric IOL, obtaining significantly better monocular and binocular UDVA with the EDOF IOL. However, these authors did not find significant differences among EDOF and monofocal groups in terms of contrast sensitivity 
$(P \geq 0.156)$ or ocular optical quality parameters $(P \geq 0.084)$. The excellent distance visual outcomes obtained with the EDOF IOL evaluated in our series are also consistent with the good level of refractive predictability, with almost all eyes having a postoperative spherical equivalent within $\pm 0.50 \mathrm{D}$.

Concerning intermediate vision, mean 1 -month postoperative logMAR DCIVA (measured at $80 \mathrm{~cm}$ ) was $0.14 \pm 0.07$, confirming the excellent performance of the EDOF IOL at intermediate vision. This was consistent with the high levels of general patient satisfaction with intermediate vision found in our study. A mean decimal UIVA of 0.80 was reported in the Concerto multicenter study evaluating the outcomes of the EDOF IOL Tecnis Symfony, with a median patient satisfaction score of 10.0 (scale, 0 to 10) [19]. Savini and coauthors [10] reported a mean value of $\log$ MAR DCIVA of $0.15 \pm 0.11$ in 20 eyes implanted with a refractive EDOF IOL based on the control of the induced spherical aberration. These levels of intermediate visual acuity achieved with the evaluated EDOF IOL are consistent or even better than those provided by trifocal diffractive IOLs [12] [13] [14]. Hamid and Sokwala [13] found significantly better UIVA outcomes with a diffractive-based EDOF IOL and two models of trifocal IOLs (AT.LISAtri and FineVision). In contrast, other studies comparing diffractive EDOF and trifocal IOLs have not shown significant differences in intermediate visual performance [12] [18]. The near visual performance was found to be more limited, as reported for other types of EDOF IOLs [9] [10] [11] [12] [13] [15]-[21]. We obtained a mean logMAR DCNVA $(40 \mathrm{~cm})$ of $0.30 \pm 0.08$. This level of near vision is lower than that provided by multifocal IOLs [9] [12] [13] [18], but it is a functional level as confirmed by the patient satisfaction questionnaire. Specifically, we obtained a medianvalue of 4.0 (scale, 0 to 5 ) for the general patient satisfaction with near vision which confirms that the level of near vision provided by the evaluated EDOF IOL is functional. Similarly, a mean decimal UNVA of 0.69 was reported in the Concerto multicenter study evaluating the outcomes of the EDOF IOL Tecnis Symfony, with a median patient satisfaction score of 8.0 (scale, 0 to 10) [19]. Savini and coauthors [10] reported a mean value of $\log$ RAD DCNVA $(40 \mathrm{~cm})$ of $0.35 \pm 0.14$ for eyes implanted with a specific model of refractive EDOF IOL.

All these visual outcomes were confirmed by the defocus curve that showed a very progressive decline of visual acuity when the level of negative defocus increased up to $-2 \mathrm{D}$. The decrease of visual acuity is significantly more pronounced for defocus levels of more than $2 \mathrm{D}$. This shape of the defocus curve has been also reported for other types of EDOF IOLs [9] [10] [12] [18] [20]. Previous comparative studies have shown that the level of near visual performance with EDOF IOLs compared to multifocal IOLs is more limited but within a functional range [9] [10] [12] [18], allowing the patient to perform daily activities without difficulties. This is reflected in the high level of patient satisfaction for near vision. Ruiz-Mesa and coauthors [12] [18] found in two comparative studies that the defocus curves were significantly better for defocus levels be- 
tween -2.50 and $-4.00 \mathrm{D}$ with trifocal IOLs than with an EDOF IOL. Pedrotti and colleagues [9] reported as well a more limited near visual outcome in eyes implanted with an EDOF IOL compared to those implanted with a high addition multifocal IOL. However, in all these previous comparative series, the general level of patient satisfaction was high, similar as in our study. Indeed, a total of $95 \%$ of patients reported that they would have the same surgical procedure with the same IOL again, with only $11 \%$ of patients reporting the occasional use of glasses for near, especially for prolonged tasks. Similarly, in the Concerto multicentre study, more than $91 \%$ of patients implanted with a diffractive EDOF IOL reported that they would recommend the same procedure to their friends and family.

The incidence of photic phenomena was minimal, with a median value of 4.0 each (scale, 0 -worst situation to 5-best situation) in the patients' evaluation of postoperative night driving and difficulties associated to halos. Likewise, a median value of 5.0 (with the same scale) was obtained for the subjective patient evaluation of the postoperative adaption between photopic and mesopic conditions. This confirms one of the potential advantages of EDOF IOLs, the generation of less disturbing photic phenomena. Hamid and Sokwala [13] reported in a comparative study that the perception of halos with a trifocal IOL was more disturbing compared to EDOF IOLs. However, other authors such as Escandón-García et al. [22] found in a prospective case series that objective dysphotopsia measured with a light distortion analyzer was not reduced in eyes implanted with EDOF IOLs compared to trifocal IOLs. Possibly, in some patients with specific characteristics, the tolerance to the double halo generated by trifocal IOLs [23] is similar to that associated to the halos of lower magnitude generated by EDOF IOLs. Future studies should investigate this further.

\section{Conclusion}

The EDOF IOL AT LARA is able to provide a restoration of the visual function across distances after cataract surgery, with very high levels of patient satisfaction. The near visual performance obtained with this IOL is more limited than that obtained with multifocal IOLs, but it is within a functional range allowing the patient to perform most of the daily activities without the need for glasses. As expected according to the design of the IOL, the incidence of photic phenomena was minimal, with low impact on night driving. Future studies should confirm all these outcomes in larger samples over a longer follow-up period.

\section{Financial Disclosure}

The study was supported by a research grant of Carl Zeiss Meditec.

\section{References}

[1] Breyer, D.R.H., Kaymak, H., Ax, T., Kretz, F.T.A., Auffarth, G.U. and Hagen, P.R. (2017) Multifocal Intraocular Lenses and Extended Depth of Focus Intraocular 
Lenses. The Asia-Pacific Journal of Ophthalmology (Phila), 6, 339-349.

[2] Yoo, Y.S., Whang, W.J., Byun, Y.S., Piao, J.J., Kim, D.Y., Joo, C.K. and Yoon, G. (2018) Through-Focus Optical Bench Performance of Extended Depth-of-Focus and Bifocal Intraocular Lenses Compared to a Monofocal Lens. Journal of Refractive Surgery, 34, 236-243. https://doi.org/10.3928/1081597X-20180206-04

[3] Alba-Bueno, F., Garzón, N., Vega, F., Poyales, F. and Millán, M.S. (2018) Patient-Perceived and Laboratory-Measured Halos Associated with Diffractive Bifocal and Trifocal Intraocular Lenses. Current Eye Research, 43, 35-42. https://doi.org/10.1080/02713683.2017.1379541

[4] de Vries, N.E., Webers, C.A., Touwslager, W.R., Bauer, N.J., de Brabander, J., Berendschot, T.T. and Nuijts, R.M. (2011) Dissatisfaction after Implantation of Multifocal Intraocular Lenses. Journal of Cataract \& Refractive Surgery, 37, 859-865. https://doi.org/10.1016/j.jcrs.2010.11.032

[5] Kamiya, K., Hayashi, K., Shimizu, K., Negishi, K., Sato, M. and Bissen-Miyajima, H.; Survey Working Group of the Japanese Society of Cataract and Refractive Surgery (2014) Multifocal Intraocular Lens Explantation: A Case Series of 50 Eyes. American Journal of Ophthalmology, 158, 215-220.

https://doi.org/10.1016/j.ajo.2014.04.010

[6] Camps, V.J., Tolosa, A., Piñero, D.P., de Fez, D., Caballero, M.T. and Miret, J.J. (2017) In Vitro Aberrometric Assessment of a Multifocal Intraocular Lens and Two Extended Depth of Focus IOLs. Journal of Ophthalmology, 2017, 7095734. https://doi.org/10.1155/2017/7095734

[7] Weeber, H.A., Meijer, S.T. and Piers, P.A. (2015) Extending the Range of Vision Using Diffractive Intraocular Lens Technology. Journal of Cataract \& Refractive Surgery, 41, 2746-54. https://doi.org/10.1016/j.jcrs.2015.07.034

[8] Bellucci, R. and Curatolo, M.C. (2017) A New Extended Depth of Focus Intraocular Lens Based on Spherical Aberration. Journal of Refractive Surgery, 33, 389-394. https://doi.org/10.3928/1081597X-20170329-01

[9] Pedrotti, E., Carones, F., Aiello, F., Mastropasqua, R., Bruni, E., Bonacci, E., Talli, P., Nucci, C., Mariotti, C. and Marchini, G. (2018) Comparative Analysis of Visual Outcomes with 4 Intraocular Lenses: Monofocal, Multifocal, and Extended Range of Vision. Journal of Cataract \& Refractive Surgery, 44, 156-167. https://doi.org/10.1016/j.jcrs.2017.11.011

[10] Savini, G., Schiano-Lomoriello, D., Balducci, N. and Barboni, P. (2018) Visual Performance of a New Extended Depth-Of-Focus Intraocular Lens Compared to a Distance-Dominant Diffractive Multifocal Intraocular Lens. Journal of Refractive Surgery, 34, 228-235. https://doi.org/10.3928/1081597X-20180125-01

[11] de Medeiros, A.L., de Araújo Rolim, A.G., Motta, A.F.P., Ventura, B.V., Vilar, C., Chaves, M.A.P.D., Carricondo, P.C. and Hida, W.T. (2017) Comparison of Visual Outcomes after Bilateral Implantation of a Diffractive Trifocal Intraocular Lens and Blended Implantation of an Extended Depth of Focus Intraocular Lens with a Diffractive Bifocal Intraocular Lens. Clinical Ophthalmology, 11, 1911-196. https://doi.org/10.2147/OPTH.S145945

[12] Ruiz-Mesa, R., Abengózar-Vela, A. and Ruiz-Santos, M. (2017) A Comparative Study of the Visual Outcomes between a New Trifocal and an Extended Depth of Focus Intraocular Lens. European Journal of Ophthalmology [epub ahead of print]. https://doi.org/10.5301/ejo.5001029

[13] Hamid, A. and Sokwala, A. (2016) A More Natural Way of Seeing: Visual Performance of Three Presbyopia Correcting Intraocular Lenses. Open Journal of Oph- 
thalmology, 6, 176-183. https://doi.org/10.4236/ojoph.2016.63025

[14] Alió, J.L., Plaza-Puche, A.B., Montalban, R. and Javaloy, J. (2012) Visual Outcomes with a Single-Optic Accommodating Intraocular Lens and a Low-Addition-Power Rotational Asymmetric Multifocal Intraocular Lens. Journal of Cataract \& Refractive Surgery, 38, 978-985. https://doi.org/10.1016/j.jcrs.2011.12.033

[15] Plaza-Puche, A.B., Alio, J.L., Sala, E. and Mojzis, P. (2016) Impact of Low Mesopic Contrast Sensitivity Outcomes in Different Types of Modern Multifocal Intraocular Lenses. European Journal of Ophthalmology, 26, 612-617. https://doi.org/10.5301/ejo.5000777

[16] Sachdev, G.S., Ramamurthy, S., Sharma, U. and Dandapani, R. (2018) Visual Outcomes of Patients Bilaterally Implanted with the Extended Range of Vision Intraocular Lens: A Prospective Study. Indian Journal of Ophthalmology, 66, 407-410.

[17] Attia, M.S.A., Auffarth, G.U., Kretz, F.T.A., Tandogan, T., Rabsilber, T.M., Holzer, M.P. and Khoramnia, R. (2017) Clinical Evaluation of an Extended Depth of Focus Intraocular Lens with the Salzburg Reading Desk. Journal of Refractive Surgery, 33, 664-669. https://doi.org/10.3928/1081597X-20170621-08

[18] Ruiz-Mesa, R., Abengózar-Vela, A., Aramburu, A. and Ruiz-Santos, M. (2017) Comparison of Visual Outcomes after Bilateral Implantation of Extended Range of Vision and Trifocal Intraocular Lenses. European Journal of Ophthalmology, 27, 460-465. https://doi.org/10.5301/ejo.5000935

[19] Cochener, B., Concerto Study Group (2016) Clinical Outcomes of a New Extended Range of Vision Intraocular Lens: International Multicenter Concerto Study. Journal of Cataract \& Refractive Surgery, 42, 1268-1275. https://doi.org/10.1016/j.jcrs.2016.06.033

[20] Pedrotti, E., Bruni, E., Bonacci, E., Badalamenti, R., Mastropasqua, R. and Marchini, G. (2016) Comparative Analysis of the Clinical Outcomes with a Monofocal and an Extended Range of Vision Intraocular Lens. Journal of Refractive Surgery, 32, 436-442. https://doi.org/10.3928/1081597X-20160428-06

[21] Kaymak, H., Höhn, F., Breyer, D.R., Hagen, P., Klabe, K., Gerl, R.H., Mueller, M., Auffarth, G.U., Gerl, M. and Kretz, F.T. (2016) Functional Results 3 Months after Implantation of an "Extended Range of Vision" Intraocular Lens. Klinische Monatsblätter für Augenheilkunde, 233, 923-927.

https://doi.org/10.1055/s-0042-104064

[22] Escandón-García, S., Ribeiro, F.J., McAlinden, C., Queirós, A. and GonzálezMéijome, J.M. (2018) Through-Focus Vision Performance and Light Disturbances of 3 New Intraocular Lenses for Presbyopia Correction. Journal of Ophthalmology, 2018, Article ID: 61675493. https://doi.org/10.1155/2018/6165493

[23] Vega, F., Alba-Bueno, F., Millán, M.S., Varón, C., Gil, M.A. and Buil, J.A. (2015) Halo and Through-Focus Performance of Four Diffractive Multifocal Intraocular Lenses. Investigative Ophthalmology \& Visual Science, 56, 3967-3975.

https://doi.org/10.1167/iovs.15-16600 